\title{
Iron Homstatin as a Marker on the Oxidative Stress in Patients for Diabetes Mellitus Type 2
}

\author{
Saada M. Ali Younis Al-Hamadany \\ mohanad.a1986@gmail.com
}

\author{
Dr. Rukzan M. Dawood Al-Saffar \\ ruzan.saffar@gmail.com
}

Department of Chemistry / College of Education for Girls

University of Mosul

\section{Dr. Layla Abdulla Mustafa}

Department of Chemistry / College of Science

University of Mosul

Layla.mustafa@gmail.com

DOI: $\underline{10.33899 / \text { edusj.2019.162954 }}$

Received
$06 / 02 / 2019$
Accepted

12 / 03 / 2019

\begin{abstract}
The study included measurement of the level of glucose, iron, ferritin, transferrin, Total iron binding capacity, Ceruloplasmin Ferroxidase activity, peroxidase activity and lipid peroxidation in the serum of people with type 2 diabetes mellitus $\left(\mathrm{T}_{2} \mathrm{DM}\right)$. The study included (90) blood samples and divided into three groups (I, II, III) each of them (30) samples, (I) control group, (II, III) Type 2 diabetes patients with complications and without of complications, respectively. The results showed a significant increase in the level of glucose, iron, ferritin and lipid peroxidation in patients with type 2 diabetes with and without complications respectively compare with control group. While a decrease was observed efficacy of peroxidase and ferroxidase in patients Compare with control group as well as decreased transferrin and total iron binding capacity.
\end{abstract}

Key word : $T_{2} D M$, ferritin, iron, ferroxidase, TIBC. 
استقلاب الحديد كمؤشر على الكرب التأكسدي لمرضى داء السكري من النوع الثاني

\author{
أ.م.د. ركزان محمود داؤود \\ ruzan.saffar@gmail.com \\ قسم الكيمياء/ كلية التربية للبنات

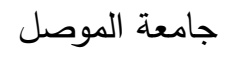

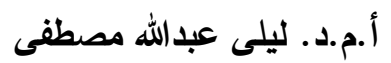 \\ قسم الكيمياء / كلية العلوم

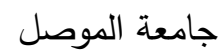

سعدة محمد علي يونس الحمداني

\title{
Layla.mustafa@gmail.com
}

DOI: $\underline{10.33899 / \text { edusj.2019.162954 }}$

القبول

2019 / 03 / 12

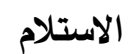

2019 / 02 / 06

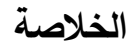

تضمنت الدراسة قياس مستوى كل من الكلوكوز والحديد والفريتين والترانسفرين وسعة ارتباط الحديد الكلية

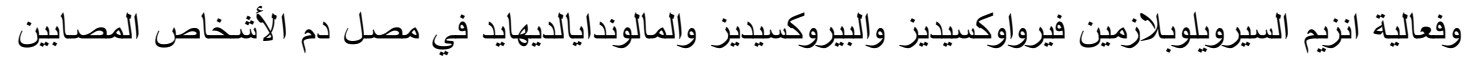
بالنوع الثاني من داء السكر • وشملت الدراسة (90) عينة دم وقسمت الى ثلاثث مجاميع لكل منهم (30)عينـة،

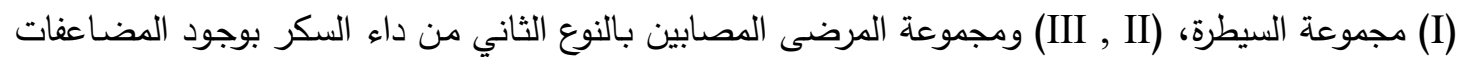

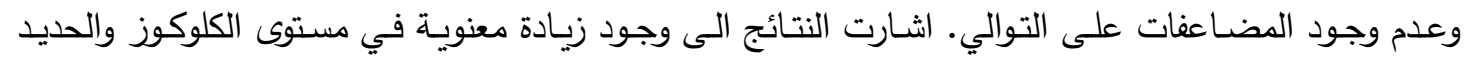

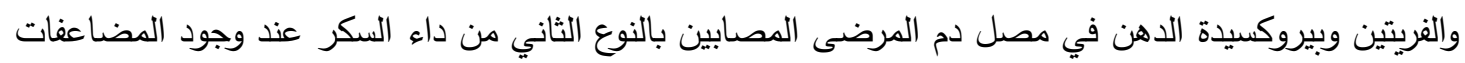

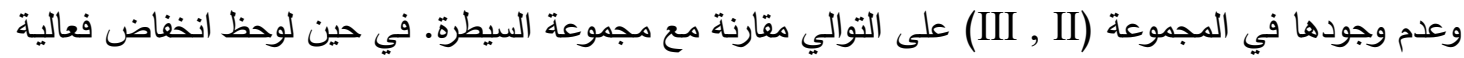

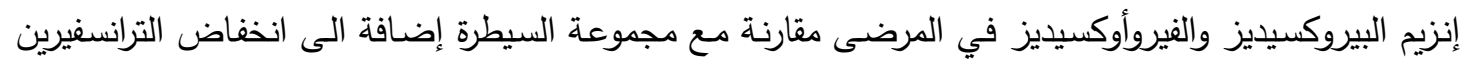
وسعة ارتباط الحديد الكلية.

الكلمات الدالة: داء السكر من النوع الثاني، الفريتين، الحديد، الفيروأوكسيديز، سعة ارتباط الحديد الكلية

\section{المقدمة Introduction}

داء السكر Diabetes mellitus : مرض مزمن يتميز بارتفاع السكر في الدم ويصاحبه اضطرابات في

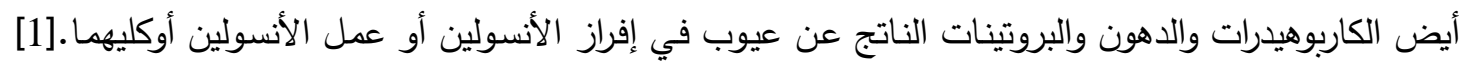

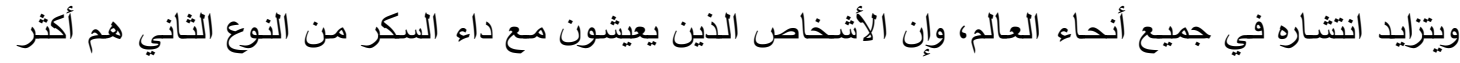

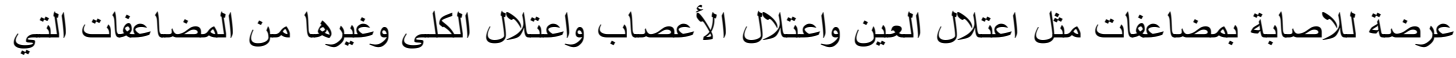
غالباً ما تؤدي إلى وفاتهم المبكرة [2]. 


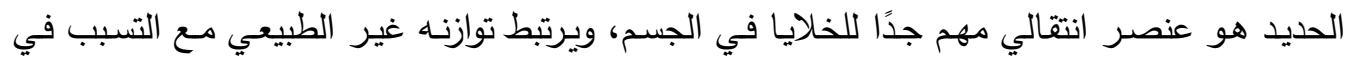

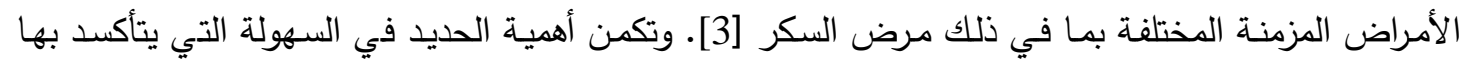

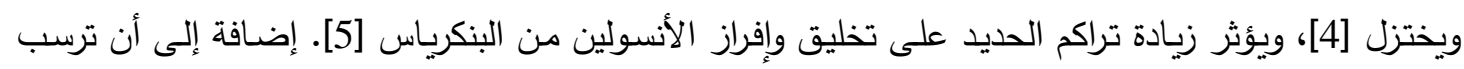
الحديد في الكبد يسبب مقاومة الأنسولين، وذلك عن طريق التحخل في قدرة الأنسولين على كبت إنتانئ إنتاج الكلوكوز

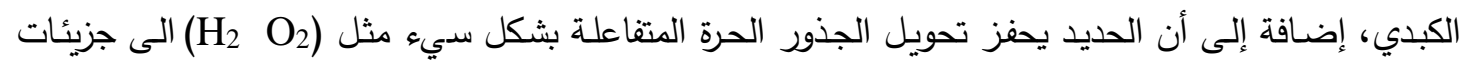

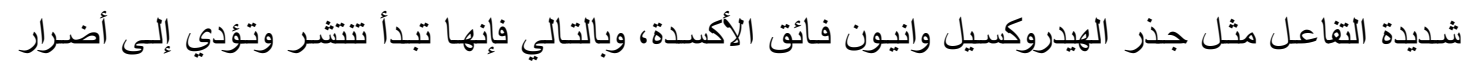
مؤكسدة]6].

الفريتين هو عبارة عن بروتين يوجد في كل مكان داخل الخلايا، ويقوم بتخزين الحديد، وهو مؤشر

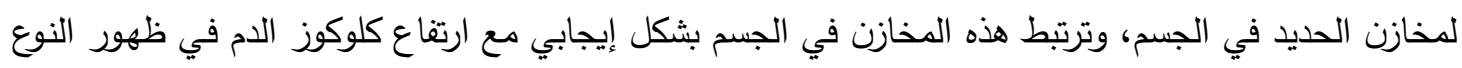
الثاني من داء السكر [7].

يتكون الفريتين من 24 وحدة فرعيـة تترتب بشكل ثلاثي الأبعاد للتخزين الديناميكي للحديد، ولهه وزن

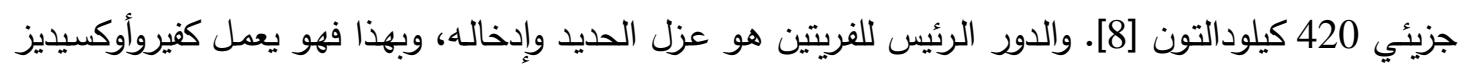
لتحويل Fe

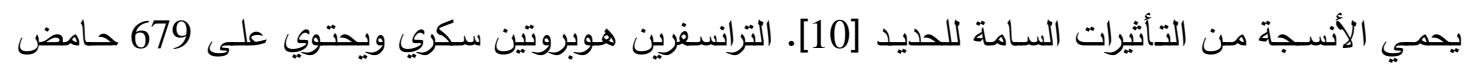

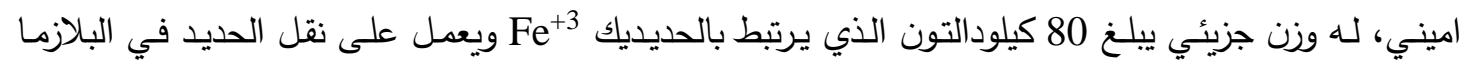

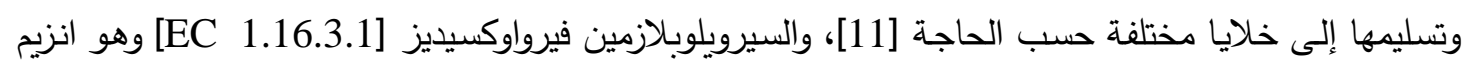

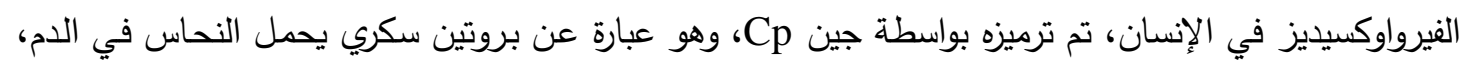

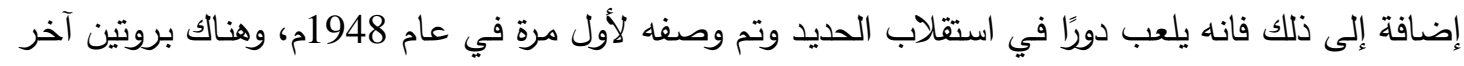

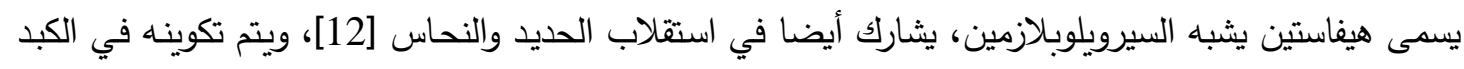

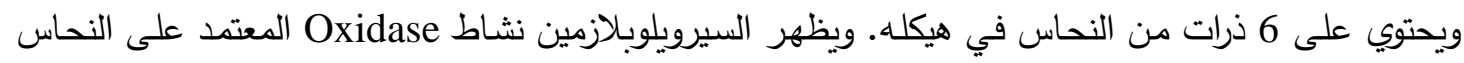

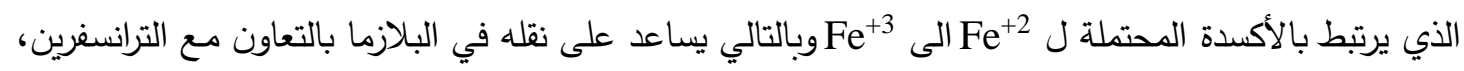

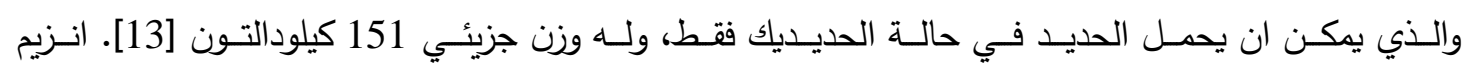

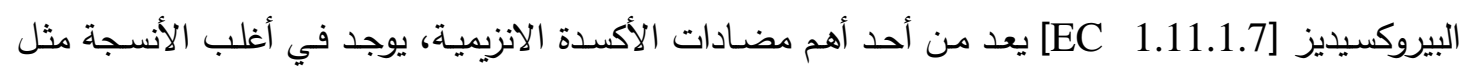

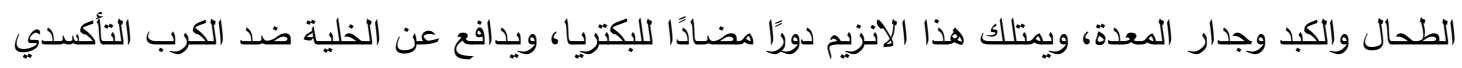

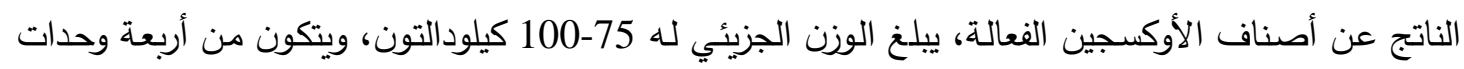

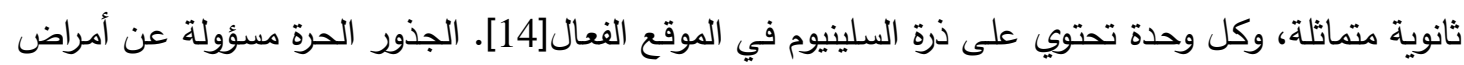

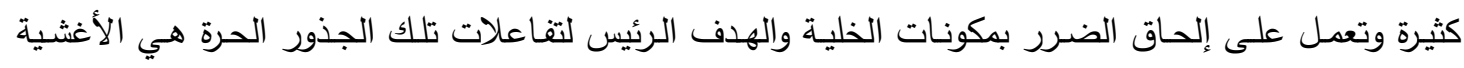

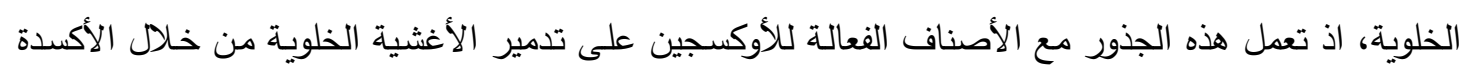

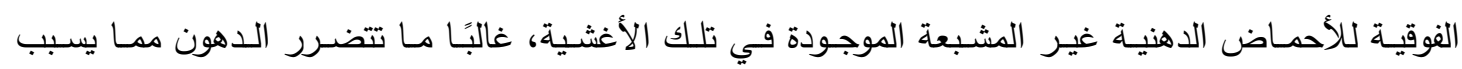

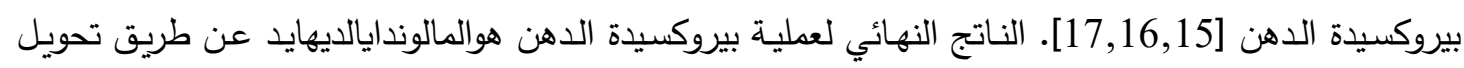

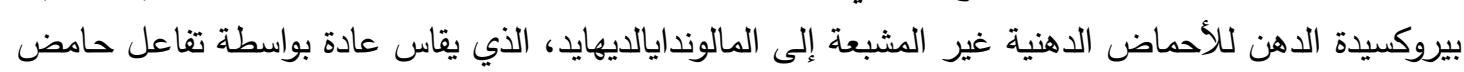
ثيوباربيتوريك، وأنه يعكس حالات زيادة توليد الجذور الحرة والكرب التأكسدي كما في داء السكر [18]. 
الهدف من البحث

دراسة استقلاب الحديد في النوع الثاني من داء السكر ، وايجاد العلاقة بين سكر الصيام والحديد والفريتين والترانسفرين والبيروكسيديز والثيرواوكسيديز وكذلك المالونالديهايد. نلاحظ من خلال ايجاد العلاقة بين المتغيرات المقاسة في المرضى مع تركيز الكلوكوزر وجود علاقة بين الحديد والكلوكوز في المرضى المصابين بداء السكر من النوع الثاني بوجود المضاعفات.

Materials and methods المواد وطرق العمل

أجريت هذه الدراسـة على (60) عينـة دم لأشخاص يعانون من داء السكر من النوع الثاني من كـال

الجنسين وبفئات عمرية مختلفة، تتراوح (28-64) سنة خلال الفترة المحصورة من أكتوبر - -2017 إلى فبراير -

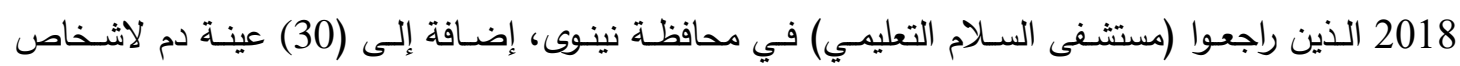
اصحاء ليكونوا كمجموعة السيطرة، وقسمت هذه العينات إلى ثلاث مجاميع:

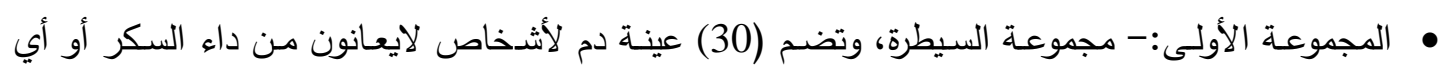

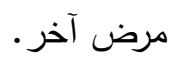
• المجموعـة الثانيـة:- وتضـــ (30) عينـة دم لأشـخاص يعـانون مـن داء السـكر مـن النـوع الثاني (بـدون مضاعفات)، وكانوا على تحكم في مستوى السكر في الدم، ومدة إصابتهم أقل من 7 سنوات.

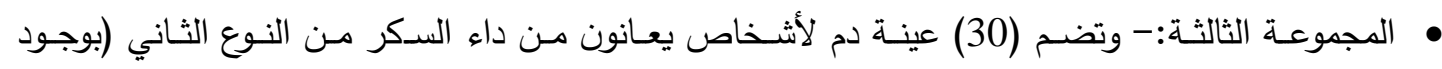
المضاعفات)، ومدة إصابتهم أكثر من 7 سنوات. تم جمع عينات الدم الوريدي في حالة الصيام لكل من مجموعتي المرضى والسيطرة، إذ تم تقدير مستوى

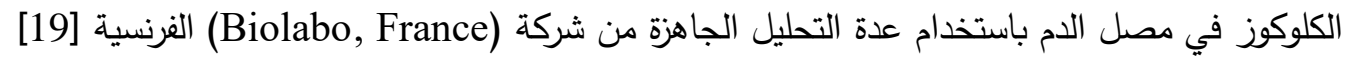
وكذلك تم تقدير مستوى كل من الحديد وسعة ارتباط الحديد الكلية في مصل الدم باستخدام عدة التحليل

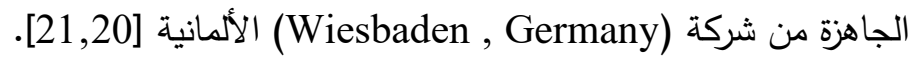
قدر مستوى الفريتين باستخدام عدة التحليل الجاهزة من شركة ( Cairo, Egypt)المصرية [22] وقياس الترانسفرين باستخدام المعادلة : Transferrin = 0.7* TIBC، وتم تقدير فعالية الفيرواوكسيديز في مصل الدام

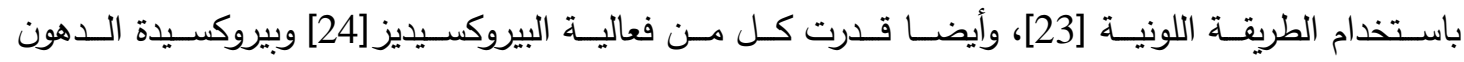
(MDA) Malondialdehyde

التحليل الإحصائي حلت نتائج مستويات كل من الكلوكوز والحديد والفريتين والترانسفرين وسعة ارتباط الحديد الكلية وفعالية

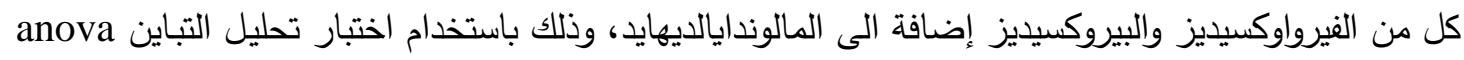

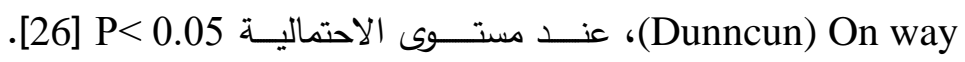

Results and discussion النتائج وإلمناقثة

أظهرت النتائج في الجدول التالي والشكل (1) أن هناك زيادة معنوية عالية في مستوى الكلوكوز لكل

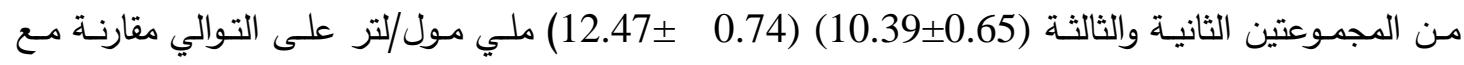




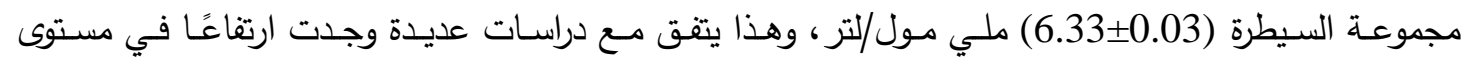

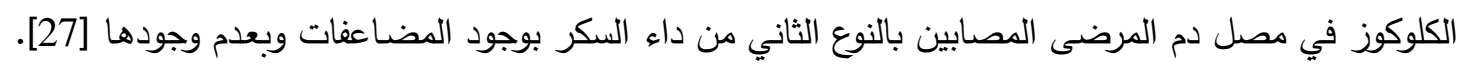
ويعود سبب الارتفاع في مستوى الكلوكوز في مصل دم المرضى المصابين بالنوع الثاني من داء السكر إلى قلى دلة إفراز الأنسولين أو خلل في مقاومة الأنسولين في الجسم، وزيادة سكر الدم لفترات طويلة في مصل دم هؤلاء

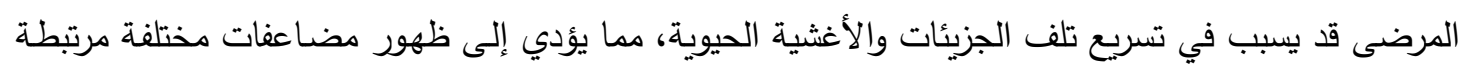
بالسكري [28].

وكذلك تشير النتائج في الجدول أعـلاه والثكل (2) إلى وجود زيـادة معنويـة في مستوى الحديد في

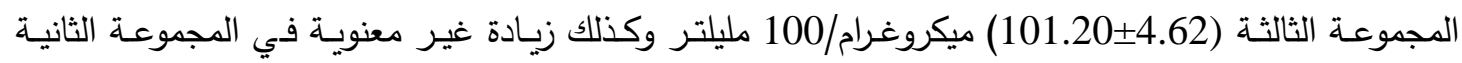

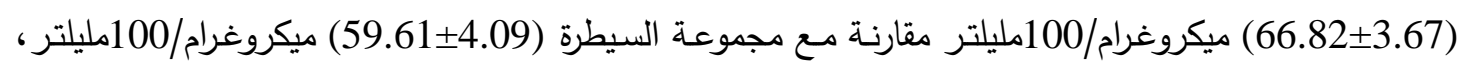

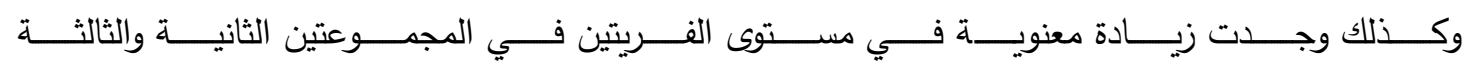

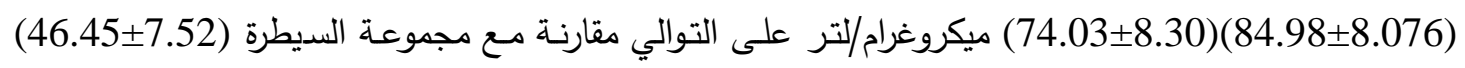

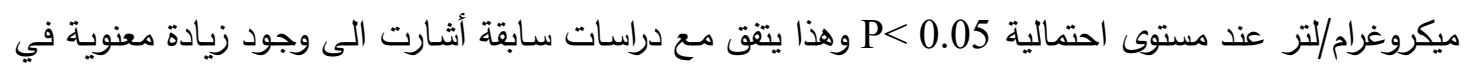

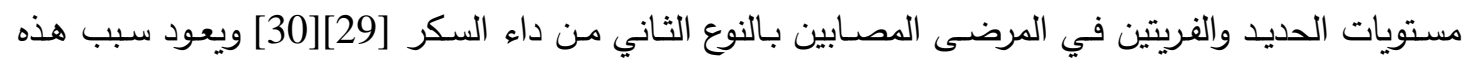

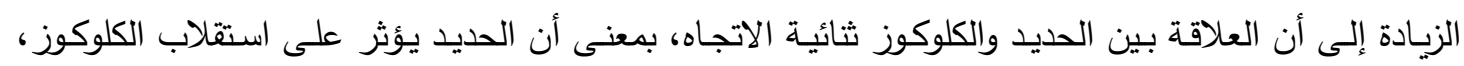
وكذلك يؤثر الكلوكوز على استقلاب الحديد، والحديد مؤكسد قوي، وقد ثبت أن أنواع الأوكسجين التفاعلية تتداخل

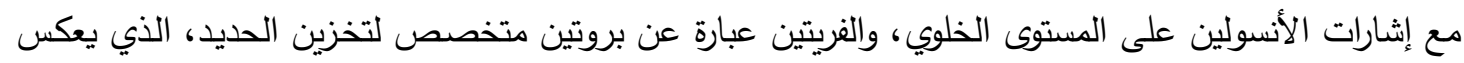

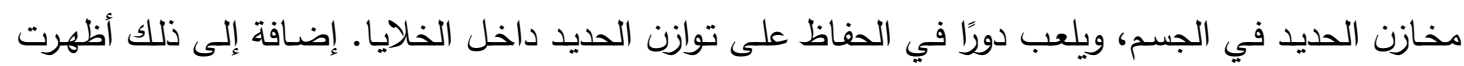

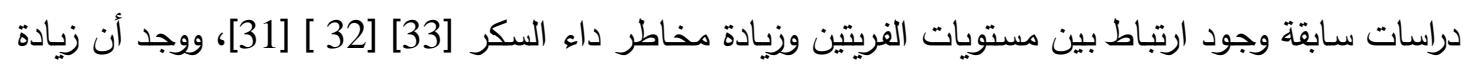

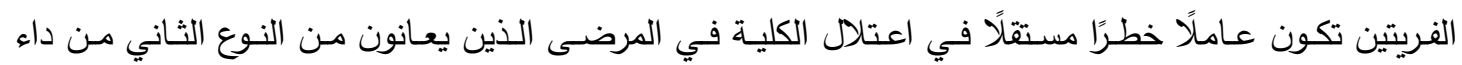
السكر [34].

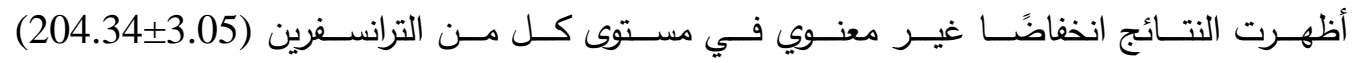

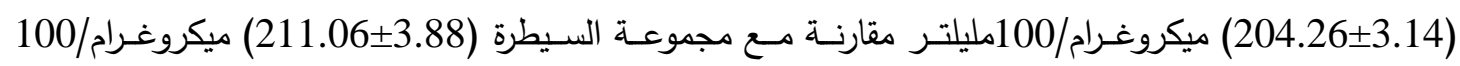

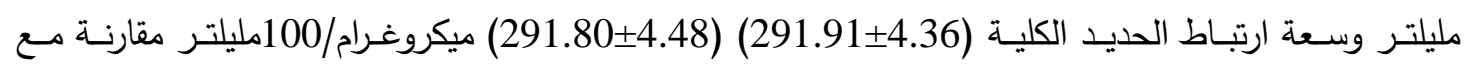

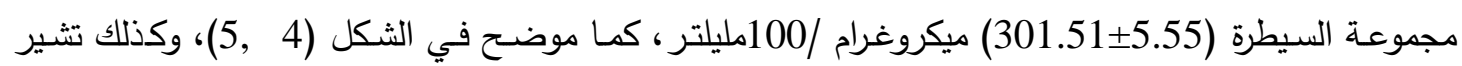

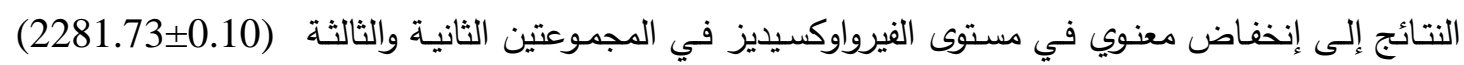

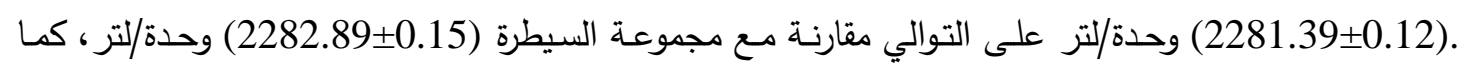
موضح في الثكل (6)، وهذا يتفق مع دراسات سابقة وجدت انخفاضًا في مستوى الفيرواوكسيديز في مصل دم دمات المرضى [35] [36]، ويعود سبب انخفاض مستويات السيرويلوبلازمين فيرواوكسيديز بسبب مضادات الأكسدة الأولية، وهذه يمكن أن تعمل عن طريق منع تثكيل جذور تفاعلية مثل البروتينات التي تتحكم في مستوى الحديد

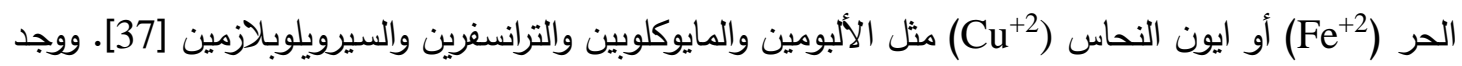

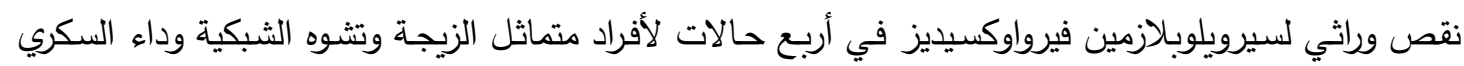
والخرف [38,39,40].

وتثير النتائج الموضحة في الجدول الى انخفاض غير معنوي في مستوى البيروكسيديز (1.22 (5.30)

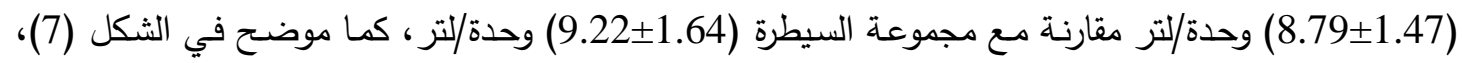
ووجود ارتفاع معنوي في مستوى المالوندايالديهايد في المجموعتين الثانية والثالثة (2.09 د 32.20 ( 30.57 ( 
1.64) نانومول/لتر على التوالي مقارنة مع مجموعة السيطرة (0.69 ـ 17.06) نانومول/لتر ، كما موضح في الثكل (8)، يعود السبب إلى حدوث بيروكسيدة الدهن وذلك بسبب زيادة الجذور الحرة الناتجة عن نشوء حالة الكرب التأكسـي المرافقـة لمرضـى داء السـكر ، بالتـالي تؤدي إلى تحطيم الأنسجة والخلايـا [41]. والإجهـاد التأكسدي يؤثر على استقلاب كل من الكلوكوز والحديد، بالتالي يؤدي إلى مقاومـة الأنسولين، ويرتبط الحديد

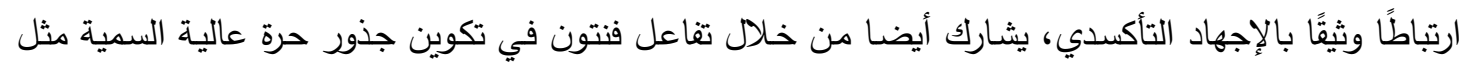

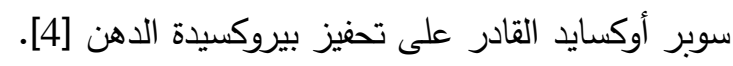

\section{الاستنتاجات Conclusions}

يستتنج مـن هذه الدراسـة بـان للحديد دوراً مهمـاً في التوازن، وبالتـالي يمكن أن تكون دراسـة الحديد والمتغيرات ذات الصلة فرعا مفيدا للدراسات التحويلية على مرض السكري ومضاعفاته، وبالتالي يمكن أن يكون تقليل الحديد الزائد المرتبط بسوء التحكم في نسبة السكر في الدم على أنه علامة متاحة لمرض السكري ليس فقط

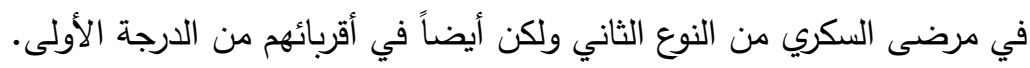

الجدول (1): مستوى المتغيرات الكيموحيوية للمجاميع الثالاثة المدرسة

\begin{tabular}{|c|c|c|c|}
\hline $\begin{array}{c}\text { مجموعة السيطرة }=0 \\
\text { Mean }=\text { SE }\end{array}$ & $\begin{array}{c}\text { مرضى بدون مضاعفات } \\
30 \text { = العدد } \\
\text { Mean I SE }\end{array}$ & $\begin{array}{c}\text { مرضى بمضاعفات } \\
\text { 30= العدد } \\
\text { Mean } \pm \text { SE }\end{array}$ & المتغيرات الكيموحيوية \\
\hline $\begin{array}{c}6.33 \pm 0.03 \\
\mathrm{a} \\
\end{array}$ & $\begin{array}{c}10.39 \pm 0.65 \\
b\end{array}$ & $\begin{array}{c}12.47 \pm 0.74 \\
\mathrm{c}\end{array}$ & mmol/L الكلوكوز \\
\hline $\begin{array}{c}59.61 \pm 4.09 \\
\mathrm{a} \\
\end{array}$ & $\begin{array}{c}66.82 \pm 3.67 \\
\mathrm{a} \\
\end{array}$ & $\begin{array}{c}101.20 \pm 4.62 \\
b\end{array}$ & الحديد Mg/dL \\
\hline $\begin{array}{c}46.45 \pm 7.52 \\
\mathrm{a} \\
\end{array}$ & $\begin{array}{c}74.03 \pm 8.30 \\
b \\
\end{array}$ & $\begin{array}{c}84.98 \pm 8.08 \\
\mathrm{~b} \\
\end{array}$ & الفريتين رg/L \\
\hline $\begin{array}{c}211.06 \pm 3.88 \\
\mathrm{a}\end{array}$ & $\begin{array}{c}204.34 \pm 3.05 \\
\mathrm{a}\end{array}$ & $\begin{array}{c}204.26 \pm 3.14 \\
\mathrm{a}\end{array}$ & الترانسفرين Mg/dL \\
\hline $\begin{array}{c}301.51 \pm 5.55 \\
\mathrm{a}\end{array}$ & $\begin{array}{c}291.91 \pm 4.36 \\
\mathrm{a}\end{array}$ & $\begin{array}{c}291.80 \pm 4.48 \\
\mathrm{a}\end{array}$ & سعة ارتباط الحديد الكلية \\
\hline $\begin{array}{c}2282.89 \pm 0.15 \\
\text { b }\end{array}$ & $\begin{array}{c}2281.73 \pm 0.10 \\
\mathrm{a}\end{array}$ & $\begin{array}{c}2281.39 \pm 0.12 \\
\mathrm{a}\end{array}$ & U/L الفيرواوكسيديز \\
\hline $\begin{array}{c}9.22 \pm 1.64 \\
\mathrm{a} \\
\end{array}$ & $\begin{array}{c}8.79 \pm 1.47 \\
\mathrm{a} \\
\end{array}$ & $\begin{array}{c}5.30 \pm 1.22 \\
\mathrm{a} \\
\end{array}$ & U/L البيروكسيديز \\
\hline $\begin{array}{c}17.06 \pm 0.69 \\
\mathrm{a}\end{array}$ & $\begin{array}{c}30.57 \pm 2.09 \\
b\end{array}$ & $\begin{array}{c}32.20 \pm 1.64 \\
\mathrm{~b}\end{array}$ & $\begin{array}{c}\text { المالوندايالديهايد } \\
\text { nmol/L }\end{array}$ \\
\hline
\end{tabular}

الأحرف الانكليزية الصغيرة المختلفة الموجودة بشكل أفقي تشير إلى اختلافات معنوية عند مستوى احتمالية (P<0.05). 


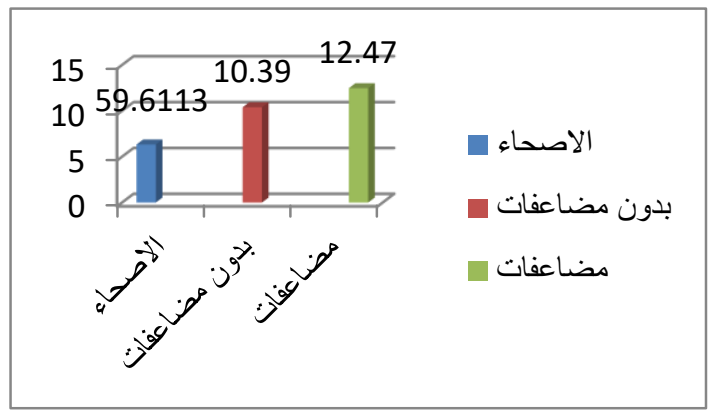

الثكل (1): مستوى الكلوكوز في المجاميع الثلاثة 
استقلاب الحديد كمؤشر على الكرب التأكسدي لمرضى داء السكري من النوع الثاني.

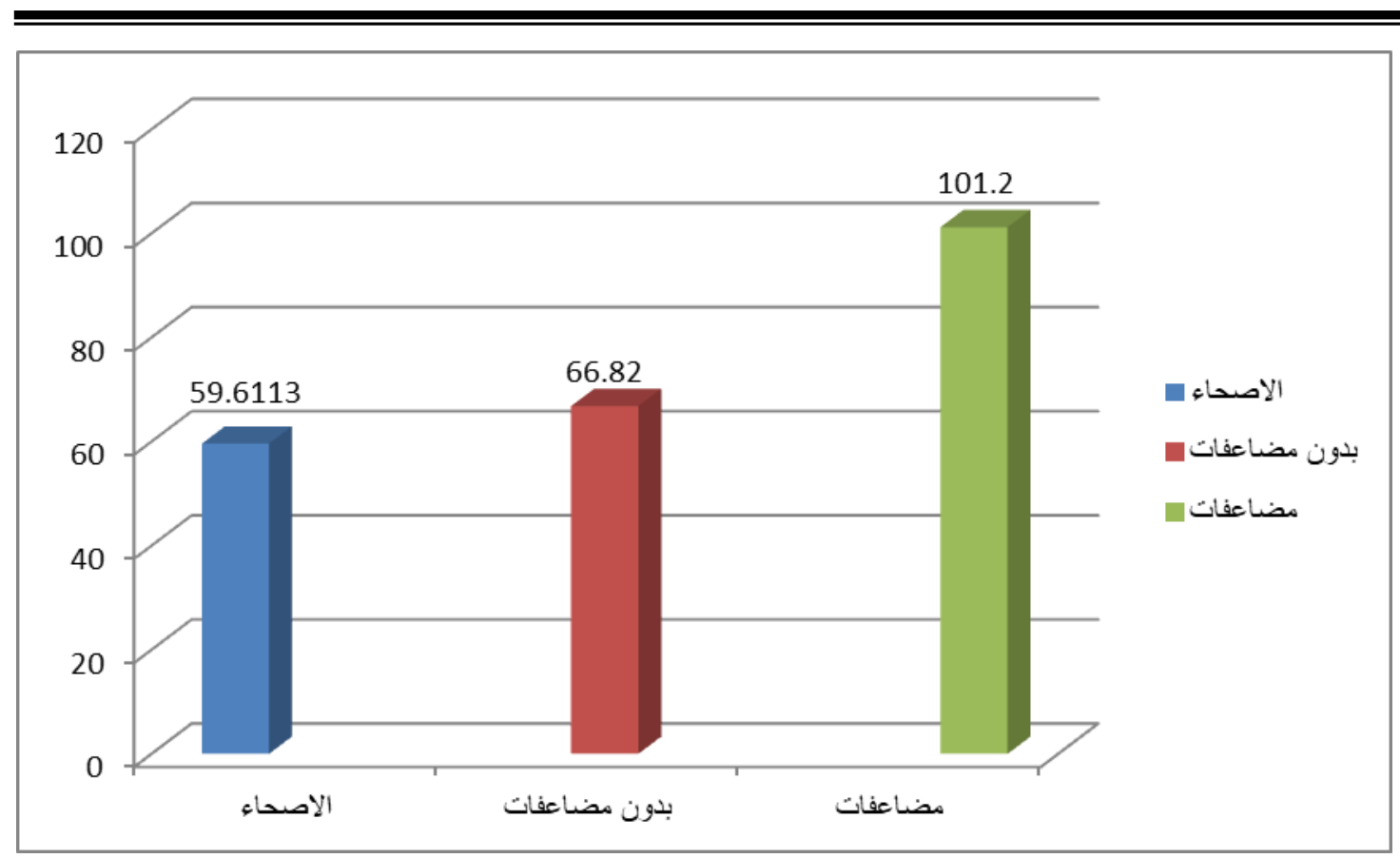

الثكل (2): مستوى الحديد في المجاميع الثلاثة

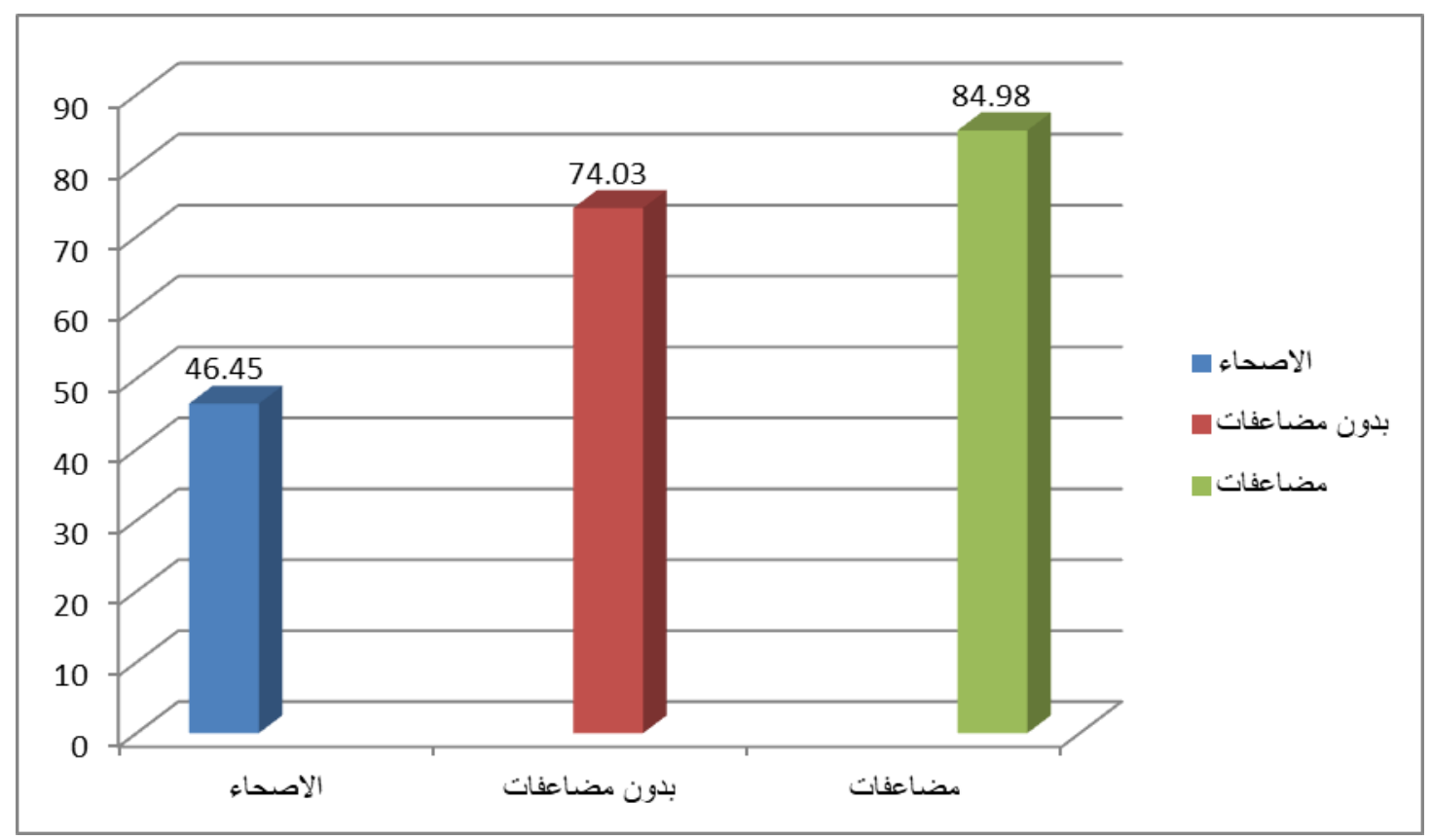

الثكل (3): مستوى الفريتين في المجاميع الثلاثة 


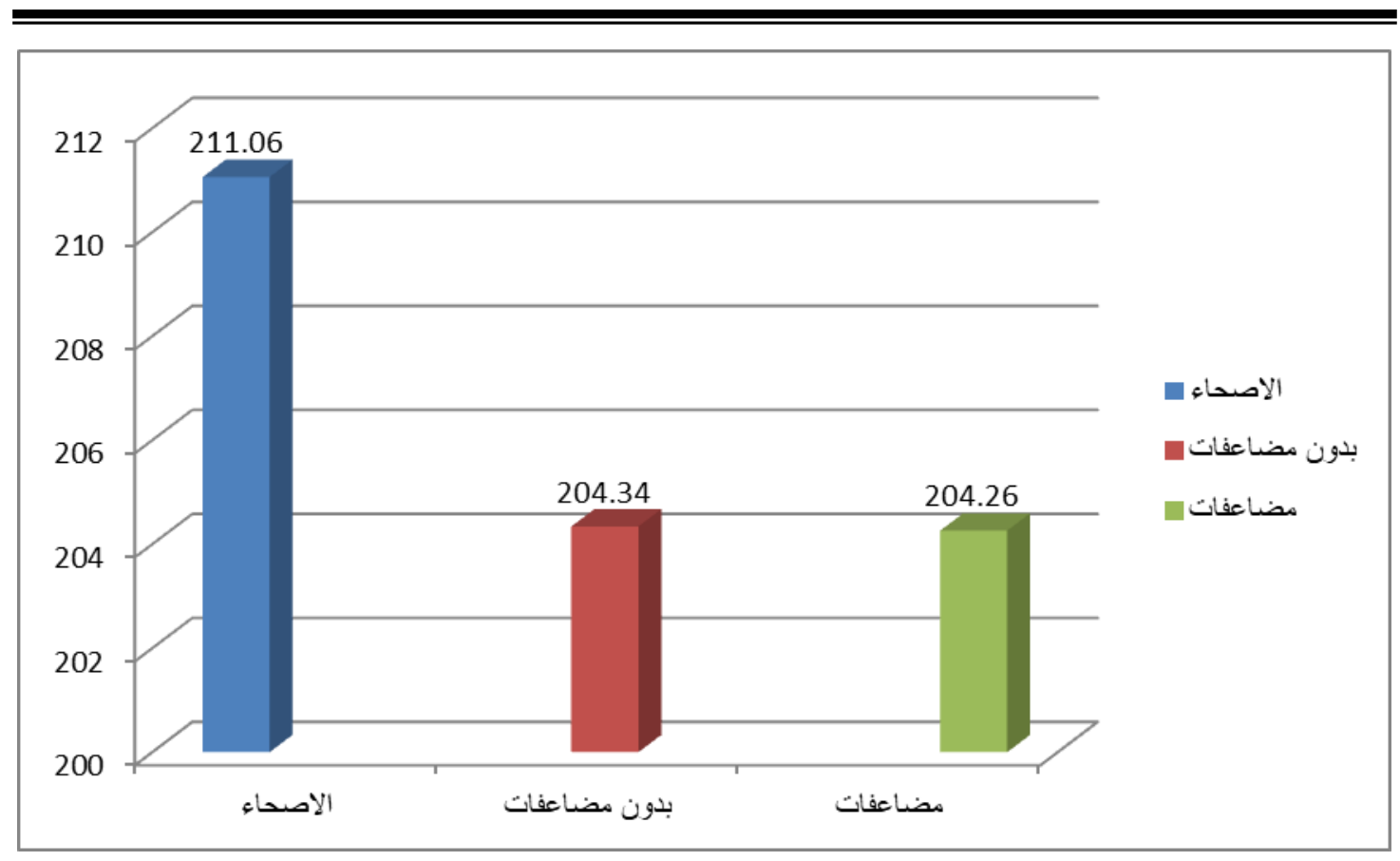

الثكل (4): مستوى الترانسفرين في المجاميع الثلاثة

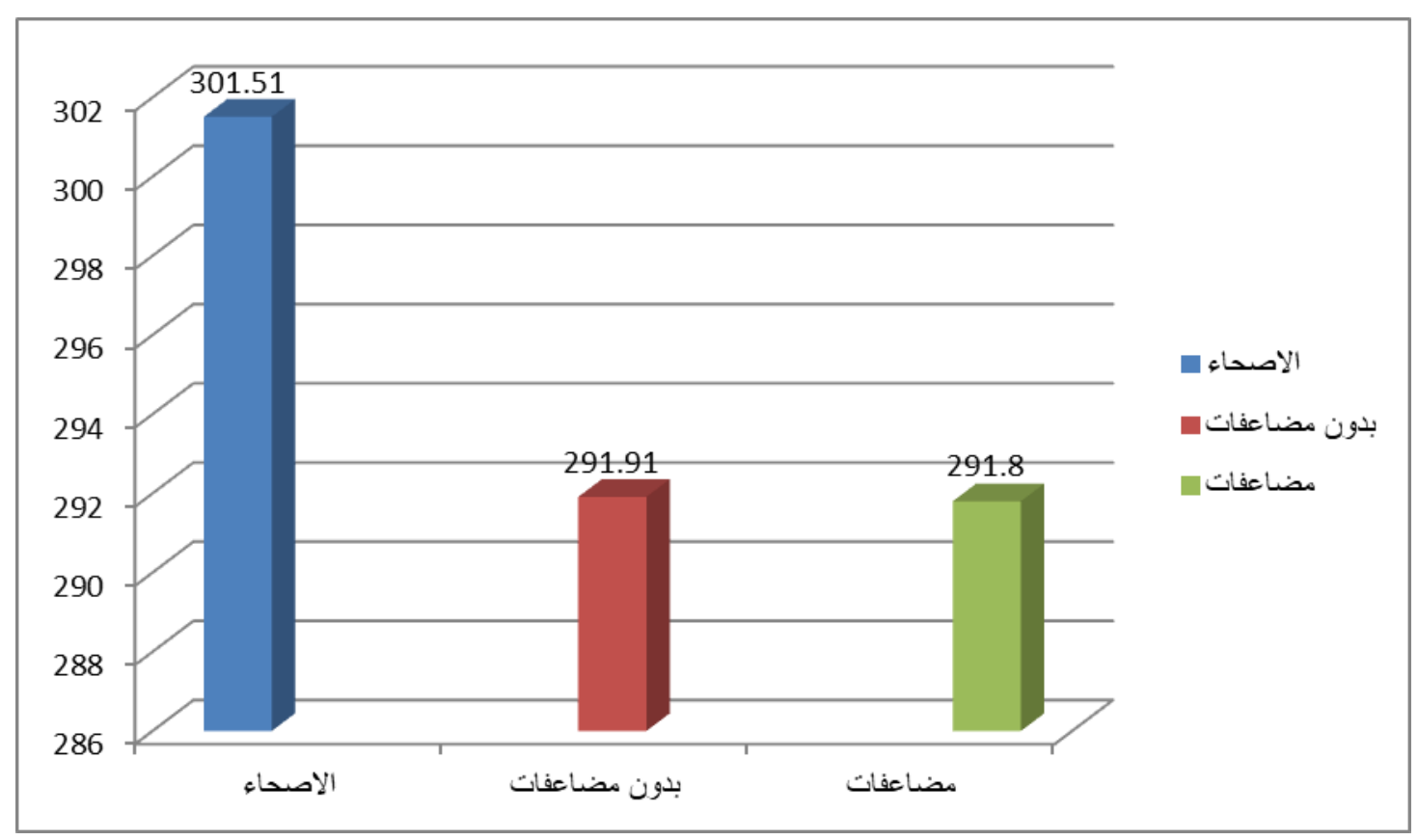

الثكل (5): مستوى سعة ارتباط الحديد الكلية في المجاميع الثلاثة 
استقلاب الحديد كمؤشر على الكرب التأكسدي لمرضى داء السكري من النوع الثاني.

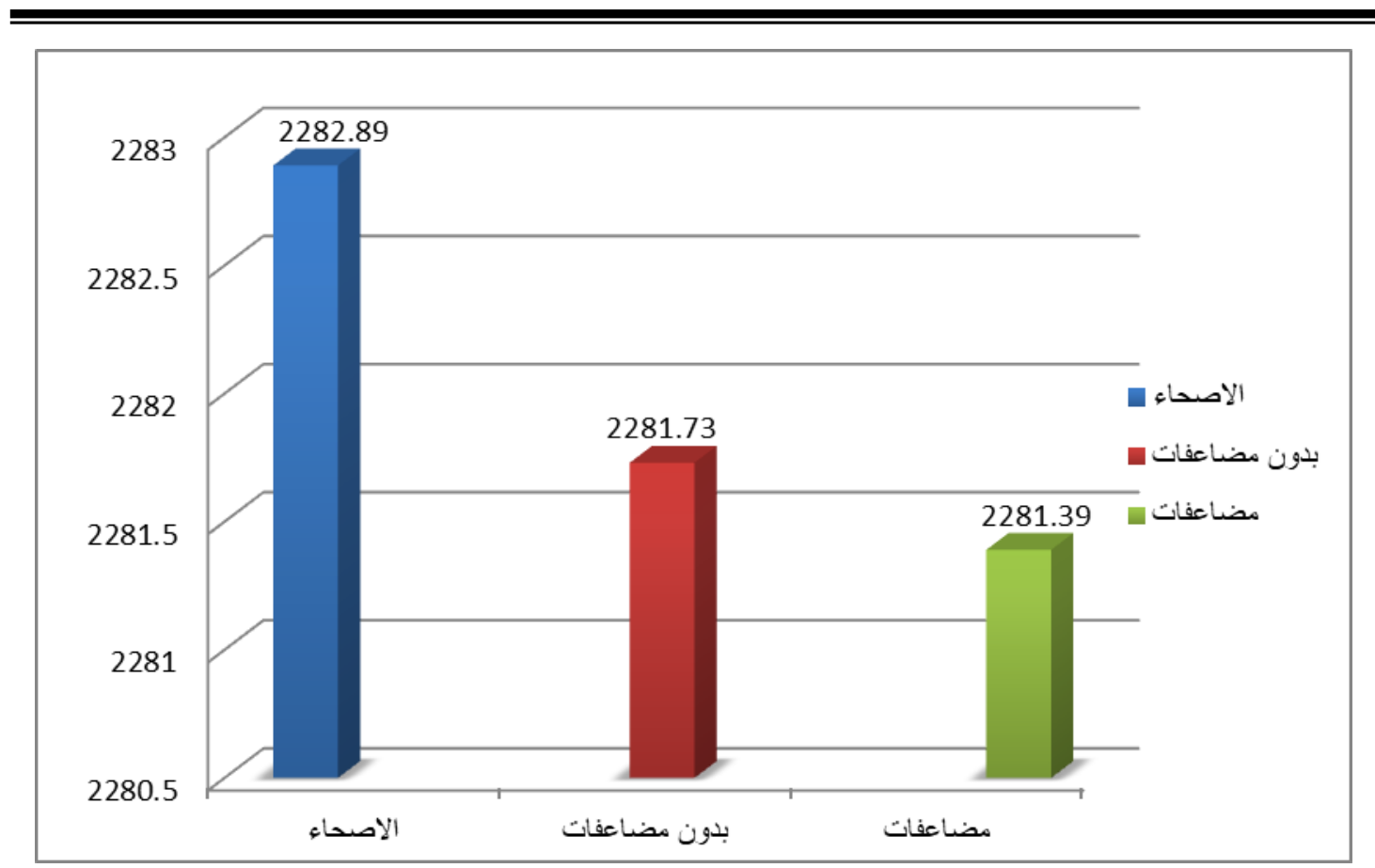

الثكل (6): مستوى الفيرواوكسيديز في المجاميع الثلاثة

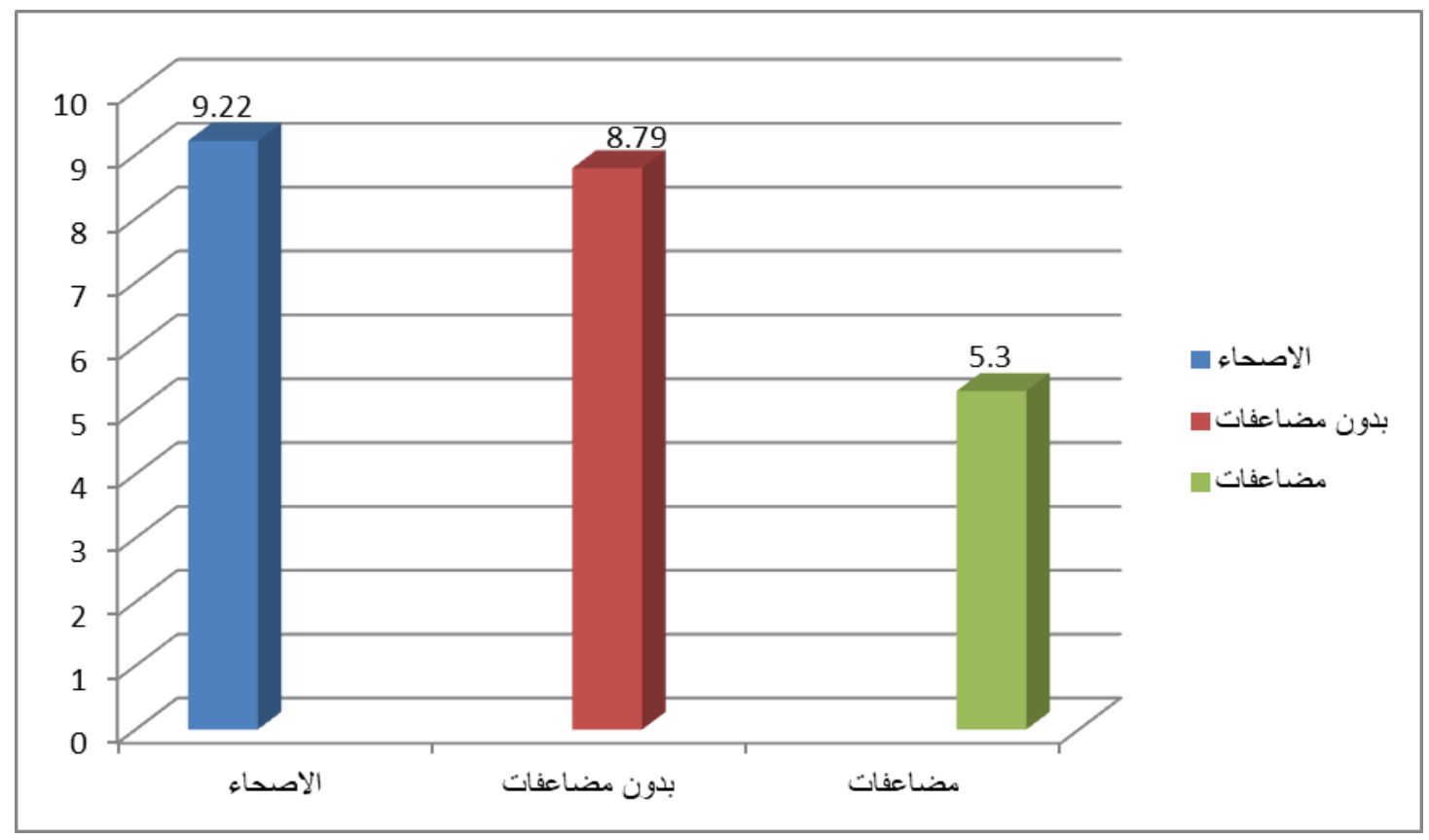

الثكل (7): مستوى البيروكسيديز في المجاميع الثلاثة 


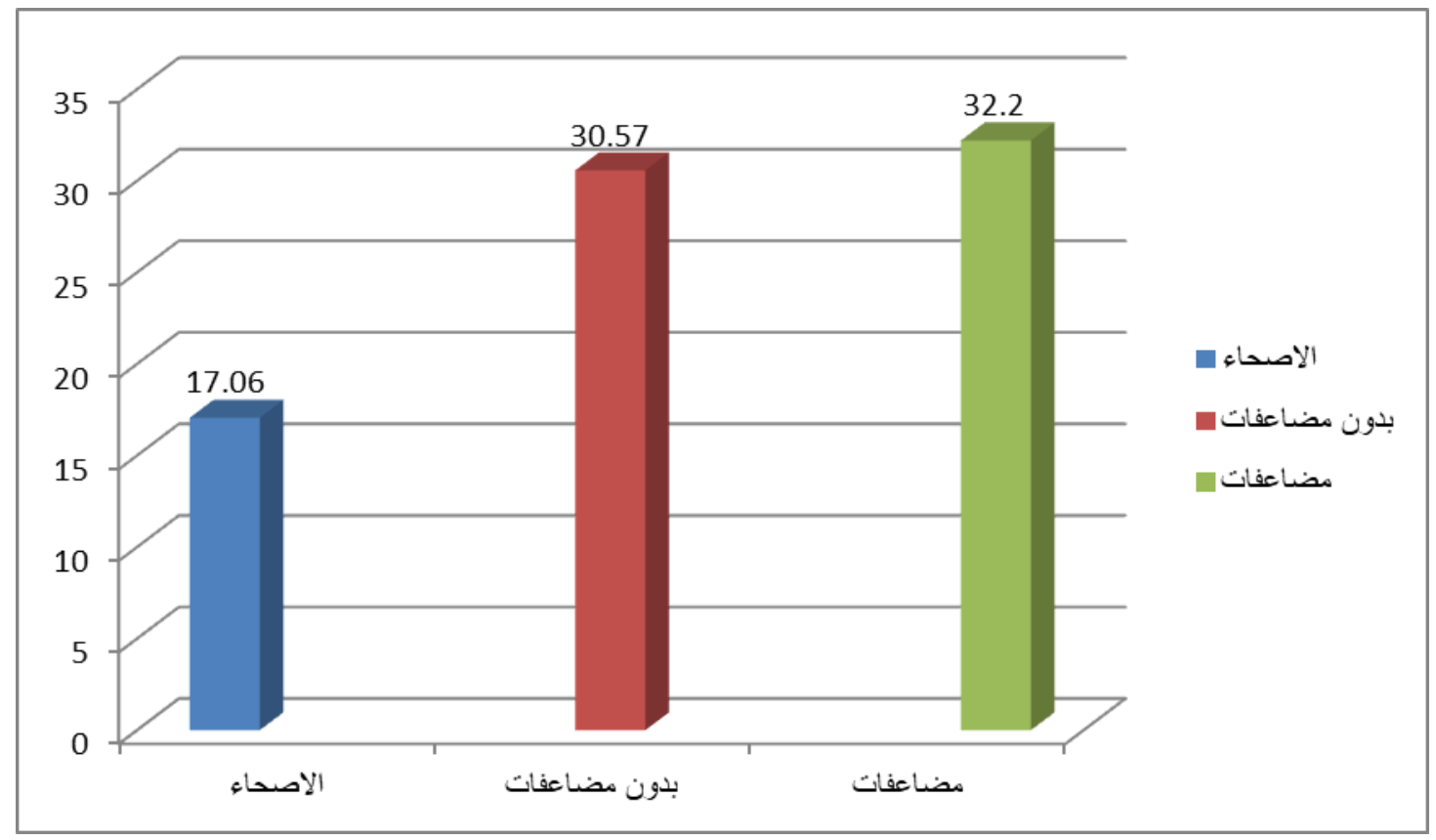

الثكل (8): مستوى المالوندايالايهايد في المجاميع الثلاثة 
References المصادر

1) Ngaski A.A., Correlation of Antioxidants Enzymes Activity With Fasting Blood Glucose in Diabetic Patients in Sokoto, Nigeria JAMMR 25(12):1-6 (2018).

2) Arora, P., Correlation between Serum Ferritin and Glycated Hemoglobin Level in Patients of Type 2 Diabetes Mellitus Original Research Article 9(6) :30-32. (2017).

3) Achuthan A and Mageswari U, Correlation between serum free iron, glycated hemoglobin and insulin resistance in uncontrolled type-2 diabetic patients, Natl J Physiology, Pharmacy and Pharmacology 7(6):642-645.(2017).

4) Gohel M., Sirajwala H.B., Chacko A., Serum Free Iron Concentration in Patients with Type 2 Diabetes Mellitus with Good and Poor Control and Its Correlation with Glycemic Control. International Journal of Diabetes Research. 2(2): 33-38.(2013).

5) Rahier JR, Loozen S, Goebbels RM. The hemochromatotic human pancreas: a quantitative immuno-histochemical and ultrastructural study. Diabetologia; 30: 5-12. (1987).

6) Walter R. M., Uriu-Hare J.Y., Olin K.L., Oster M.H., Anawalt B.D., Critchfield J.W., Keen C.L. Copper, zinc, manganese, and magnesium status and complications of diabetes mellitus. Diabetes Care.;14(11):1050-6. (1991).

7) Vashchenko G. and Ross T. A. MacGillivray Multi-Copper Oxidases and Human Iron Metabolism Nutrients 5, 2289-2313 (2013).

8) Finazzi, D. \& Arosio, P. Biology of ferritin in mammals: an update on iron storage, oxidative damage and neurodegeneration. Archives of Toxicology; 88:1787- 1802. (2014).

9) Knovich, M.A., Storey, J.A., Coffman, L.G., Torti, S.V. \& Torti, F.M. Ferritin for the clinician. Blood Reviews;23:95-104. (2009).

10) Marshall, W.J., Bangert, S.K. \& Lapsley, M. Disorder of haemoproteins, porphyrins and iron. Clinical Chemistry, eds. Bowes Jeremy., McMurray Carole. \& A. Collett, $7^{\text {th }}$ edn, Mosby Elsevier:289. (2012a).

11) Luck, A.N. \& Mason, A.B. Transferrin-mediated cellular iron delivery. Current Topics in Membranes;69:3-35. (2012).

12) Osaki S., Kinetic studies of ferrous ion oxidation with crystalline human ferroxidase (ceruloplasmin).J.Biol. Chem. 241(21):5053-9.(1966).

13) Osaki S., Walaas O., Kinetic Kinetic studies of ferrous ion oxidation with crystalline human ferroxidase.II. Rate constants at various steps and formation of a possible enzyme-substrate complex.J.Biol.Chem. 242 (11):2653-7.(1967).

14) Youngping B., Gray w., Selenium dependent glutathione peroxidase pro In Nature Sci 10: 321-330.(2000).

15) Matkovics A., An overview of free radical research Acta. Biol. Szeged. 47(1-4): 93-97.(2003).

16) Dubey N. K., Yadav P., Dutta A.K., Kumar V., Ray G.N. and Batra S., Free oxygen radicals in acute renal failure. India Pediatrics, 37:153-158.(2000).

17) Gupta S., and Deshmuk U., formation and function of free radicals in human body Ann Nat.Acod.Med. Sci(India), 30(1):45-54. (1994). 
18) Benge J.A. and Aust S.D., Estimation of serum Malondialdehyde level.Methods.in Enzymology, Academic press. London.51:302. (1978).

19) Burtis C.A and Ashwood E.R., Tietz Text Book of Clinical Chemistry.W.B. Saunder Company, philadel phia.pp: 490. (1999).

20) Callahan J.H., Cook O., Anal.Chem., 54: 59-62. (1982).

21) Starr R.T., Clin. Chem., 26(1): 156. (1980).

22) MilesLEal et al., Measurement of serum ferritin by a 2-site immunoradiometric assay.Anal.Biochem.,61:209-224. (1974).

23) Erel O., Automated measurement of serum peroxidase activity.Clin.Chem., 44 (11): 2313-2319.(1998).

24) Trinder P., Determination of glucose in blood using Glucose Oxidase with an altemative oxygen acceptor. Am.Clin.Biochem., 6:24.(1966).

25) Bakan E.,Taysi S., Polat M.F., Dalga S., Umudum Z., Bakan N.\&Gumus M., Nitric Oxide levels and lipid peroxidation in plasma of patients with gastric cancer Japanese Journal of Clinical Oncology, 32:162-166.(2002).

26) Kirkwood B.R., Essentials of Medical statistics ,Black well scientific Publication , U.K., PP.33-41.(1988).

27) Habib P., Samir S., Peroxidase Activity and Other Biochemical Parameters in Female with Type 2 Diabetes Miletus with and without Coronary Arteriosclerosis International Journal of Applied Science and Technology; 4(3):250-254.(2014).

28) Suvarna R., Rao S.S.,Joshic. kedage V.,Muttigi M.S., Shetty J.K. and prakash M., Paraoxonase activity in type2 Diabetes Mellitus patients with and without complications, Journal of clinical and Diagnostic Reserach.5(1):63-65.( 2011).

29) Ghosh D., Fariduddin M., Jahan S., Hasan M., Sultana N., Rahman M.A., Hasan K., Islam M., Atikur-Rahman M and Hasanat MA., Serum Ferritin in Newly Detected Diabetes and Prediabetes. Diabetes Obes Int J, 2(3): 000158.(2017).

30) Leticia O.I., Ifeanyi O.E., Queen E., Chinedum O.K., Determination of Ferritin Level and Total Iron Binding Capacity in Pregnancy and Postpartum Subjects in Owerri. IOSR Journal of Dental and Medical Sciences.13(9): 70-73.(2014).

31) Qian M, Liu M, Eaton JW. Transition metals bind to glycated proteins forming redox active 'glycochelates'. implications for the pathogenesis of certain diabetic complications. Biochem Biophys Res Commun; 250: 385-9.(1998).

32) Andrews, N.C., "Disorders of iron metabolism", The New England journal of medicine, vol. 341, no. 26, pp. 1986-1995.(1999).

33) Abd El-Halim, S.S and Lobna M. El-Hadidy, L.M. The Relationship between Serum Ferritin and Glycosylated Hemoglobin in Adults with Type 2 Diabetes Mellitus The Egyptian Journal of Hospital Medicine Vol. 64, Page 405- 410. (2016).

34) Ciudin A., Hernandez C., Simo R., Iron overload in Diabetic Retinopathy: A Cause or a Consequence of Impaired Mechanism? Experimental Diabetes Research, Article ID 714108, 8 pages, (2010).

35) Paul J.L., Sall N. D., Soni T., Poignet J. L., Lindenbaum A., Man N. K., Moatti N. \& Raichvarg D., Lipid peroxidation abnormalities in hemodialyzed patients. Nephron.64 (1): 106-109. (1993). 
36) AL-Emami S.A., and Hasan H., Phosphatases and Osteodystrophy in Chronic Renal Failure as Related to Oxidative Stress. B.Sc., M.Sc. College of Science. University of Baghdad.(2007).

37) Stahl W., Sies H., Antioxidant Defense : Vitamin E and C and Carotennoids. Diabetes, 46(2): 514-517. (1997).

38) Miyajima. H., Nishimura. Y., Mizoguchi. K.. et al.: Familial apo-Ceruloplasmin deficiency associated with blepharospasm and retina degeneration. Neurology. 37: 761 - 767. (1987).

39) Okamoto. N., wada. S.. Oga. T.. et al.: Hereditary ceruloplasmin deficiency with hemosiderosis. Hum. Genet.. 97 : 755 - 758. (1996).

40) Logan, J.I., Harveyson, KB, Wisdon.G.B., et al.:Hereditary caeruloplasmin deficiency, dementia and diabetes mellitus. Q. J. Med..87: 663-670.(1994).

41) El-Sayed E.M., Salem O., Aly H., and Mansur A.M., Potential anti diabetic and hypolipidemic effects of propolis extrac in streptozotocin - induced diabetic rats pak. J. Pharm. Sci. 22(2): 168-174.(2009). 